University of Cambridge \& Cambridge University Hospitals NHS Trust pfc25@medschl.cam.ac.uk Cite this as: $B M J 2021 ; 375: n 2683$ http://dx.doi.org/10.1136/bmj.n2683 Published: 04 November 2021

\section{Shortening the diagnostic odyssey-the impact of whole genome sequencing in the NHS}

\author{
Patrick F Chinnery professor of neurology and honorary consultant neurologist
}

I met my first patient with suspected mitochondrial disease in 1995 as a junior neurology trainee in Newcastle upon Tyne. At the time, mitochondrial disorders were thought to be exceptionally rare, with the first genetically defined causes reported only seven years earlier. ${ }^{12}$ Most genetic investigations were carried out in unregulated university laboratories located in a small number of locations worldwide, and referrals were mainly from tertiary centres after a protracted series of clinical opinions and investigations. Many clinicians had not even heard of mitochondrial diseases.

Over the next 10 years it became clear that mitochondrial disorders affect around one in 5000 people, ${ }^{3}$ placing them among the most common inherited diseases. However, their presentation varies enormously between family members, making them difficult to recognise in the clinic, and the underlying genetic basis is equally complex. In the absence of a cure, a molecular genetic diagnosis is even more important. For some mitochondrial disorders, the same clinical presentation can be inherited as an autosomal recessive trait, an autosomal dominant trait, or be passed on down the female (but not male) line-with each having very different recurrence risks, complications, and prognoses. Knowing the precise cause enables reliable family planning and prenatal diagnosis and facilitates the best supportive care through targeted surveillance for known complications.

Diagnostic services improved dramatically in 2006 with the establishment of a nationally commissioned NHS diagnostic service for suspected mitochondrial diseases based in London, Newcastle, and Oxford. Initially, the laboratories focused on mitochondrial DNA, but over the next decade, the number of different genetic causes of mitochondrial disease increased markedly. A growing number of newly recognised nuclear gene defects were found in only one or two families globally. ${ }^{4}$ These were not easily detectable using techniques available at the time, and for many, a diagnosis relied on a motivated research group using research tools to pinpoint the genetic cause, often involving international collaborations on an ad hoc basis.

Moreover, although free at the point of care, the nationally commissioned diagnostic service usually required families to travel long distances to one of the three national centres-with the travel costs not reimbursed by the NHS. This meant that people who could not afford the travel would not benefit from the same standard care as those who could. Families and clinicians needed a more comprehensive diagnostic approach encompassing the vast range of different genetic mechanisms found throughout the nuclear genome and the mitochondrial genome, with equitable access across the country. The launch of the 100 ooo Genomes Project in 2012 provided this opportunity through whole genome sequencing.

Initially piloted in three centres, and subsequently rolled out across England, the 100 ooo Genomes Project was open to referrals across the country for patients with a range of suspected rare genetic diseases, ${ }^{5}$ including mitochondrial disorders. We studied people (doi:10.1136/bmj-2021-066288) referred between 2015 and 2018 who had already undergone extensive NHS investigations for mitochondrial disease. ${ }^{6}$ We discovered the genetic cause in a further $31 \%$ of families. Looking back at all the known genetic causes of mitochondrial disorders, ${ }^{7}$ including those routinely tested in NHS laboratories, whole genome sequencing has the potential to diagnose around $90 \%$ of known mitochondrial diseases, ${ }^{8}$ and the test can be initiated by local diagnostic services, democratising access to a state of the art diagnostic approach.

We also found that whole genome sequencing identifies potentially treatable causes not considered by the referring clinicians. In the past, each one of these disorders would have required a specific diagnostic test, so a clinician had to recognise the possibility of each inherited disorder on clinical grounds to initiate specific investigations. This is not essential with whole genome sequencing, which can identify the genetic basis for almost all known inherited disorders. When a diagnosis is not possible, whole genome sequencing also provides a rich source of data to discover new genetic diseases that can be re-evaluated as new knowledge emerges.

Patients and families with rare diseases often describe a prolonged diagnostic odyssey involving multiple consultations by different specialist clinicians resulting in a series of diagnostic tests ${ }^{9}$ : these are often repeated over time and can be invasive or carry their own complications. Whole genome sequencing will shorten this diagnostic ordeal, reduce the need for intrusive investigations, and is highly likely to reduce NHS costs overall. If centralised across the whole of the UK, whole genome sequencing would establish cohorts of patients who may wish to join clinical trials on a national basis, providing hope of a treatment for these disorders that were once considered so rare as to be untreatable.

\section{Competing interests: see linked Research (doi:10.1136/bmj-2021-066288).}

Wallace DC, Singh G, Lott MT, etal. Mitochondrial DNA mutation associated with Leber's hereditary optic neuropathy. Science 1988;242:1427-30. doi: 10.1126/science.3201231 pmid: 3201231

2 Holt IJ, Harding AE, Morgan-Hughes JA. Deletions of muscle mitochondrial DNA in patients with mitochondrial myopathies. Nature 1988;331:717-9. doi: 10.1038/331717a0 pmid: 2830540 


\section{OPINION}

3 Chinnery PF, Johnson MA, Wardell TM, etal. The epidemiology of pathogenic mitochondrial DNA mutations. Ann Neurol 2000;48:188-93.

doi: 10.1002/1531-8249(200008)48:2«188::AID-ANA8〉3.0.CO;2-P pmid: 10939569

4 Schon KR, Ratnaike T, van den Ameele J, Horvath R, Chinnery PF. Mitochondrial Diseases: A Diagnostic Revolution. Trends Genet 2020;36:702-17.

doi: 10.1016/j.tig.2020.06.009 pmid: 32674947

5 Turro E, Astle WJ, Megy K, etalNIHR BioResource for the 100,000 Genomes Project.

Whole-genome sequencing of patients with rare diseases in a national health system. Nature 2020;583:96-102. doi: 10.1038/s41586-020-2434-2 pmid: 32581362

6 Schon KR, Horvath R, Wei W, etal. Use of whole genome sequencing to determine genetic basis of suspected mitochondrial disorders: cohort study. BMJ 2021;375:e066288. doi: 10.1136/BMJ-2021-066288.

7 Gorman GS, Schaefer AM, Ng Y, etal. Prevalence of nuclear and mitochondrial DNA mutations related to adult mitochondrial disease. Ann Neurol 2015;77:753-9. doi: 10.1002/ana.24362 pmid: 25652200

8 Raymond FL, Horvath R, Chinnery PF. First-line genomic diagnosis of mitochondrial disorders. Nat Rev Genet 2018;19:399-400. doi: 10.1038/s41576-018-0022-1 pmid: 29789687

9 Grier J, Hirano M, Karaa A, Shepard E, Thompson JLP. Diagnostic odyssey of patients with mitochondrial disease: Results of a survey. Neurol Genet 2018;4:e230.

doi: 10.1212/NXG.0000000000000230 pmid: 29600276 